The Emerging Neurobiology of Mindfulness and Emotion Processing

\author{
W. Michael Sayers ${ }^{\mathrm{a}}$, J. David Creswell ${ }^{\mathrm{b}}$, \& Adrienne Taren ${ }^{\mathrm{a}}$
}

${ }^{a}$ University of Pittsburgh

${ }^{\mathrm{b}}$ Carnegie Mellon University

Handbook of Mindfulness and Self-Regulation

Please address correspondence to: J. David Creswell, Assistant Professor in Psychology, Carnegie Mellon University, 5000 Forbes Ave, Pittsburgh, PA; creswell@cmu.ed 


\begin{abstract}
Mindfulness is associated with reduced negative affective states, increased positive affective states, and reduced clinical affective symptomatology (e.g., depression, anxiety) in previous studies. This chapter examines an emerging body of fMRI and EEG research exploring how mindfulness alters neurobiological emotion processing systems. We examine how dispositional (trait) mindfulness and how adopting a mindful attentional stance (after varying levels of mindfulness training) relate to changes in neural responses to affective stimuli. Evidence suggests mindfulness-related changes in a ventral affective processing network associated with core affect, a dorsal processing network associated with making attributions and appraisals of one's affective experience, and regulatory networks involved in modulating affective processes. These neural effects may underlie the previously observed relationships between mindfulness and changes in reported emotion processing and reactivity. Findings are discussed in light of existing neurobiological models of emotion and we describe important questions for the field in the coming years.
\end{abstract}




\section{The Emerging Neurobiology of Mindfulness and Emotion Processing}

An emerging body of research suggests that mindfulness is associated with self-reported and clinically relevant changes in emotion processing. Self-report measures of dispositional mindfulness are associated with reduced negative affective states and positively associated with positive affective states and traits (Brown, Ryan, \& Creswell, 2007). Moreover, mindfulnessbased interventions reduce depressive symptomatology and depression-relapse in at-risk patients (Hofmann, Sawyer, Witt, \& Oh, 2010; Teasdale et al., 2000), anxiety symptoms (Hofmann, Sawyer, Witt, \& Oh, 2010; Kabat-Zinn et al., 1992; Roemer, Orsillo, \& Salters-Pedneault, 2008), and affective disturbances in chronic pain patients (Grossman, Tiefenthaler-Gilmer, Raysz, \& Kesper, 2007; Kabat-Zinn, 1982). This body of work suggests that mindfulness may be associated with changes in emotion processing, and in this chapter we consider the extant mindfulness $\mathrm{fMRI}$ and EEG research to better understand how the brain processes affective stimuli in relation to trait mindfulness, while adopting a mindful attentional stance, and after mindfulness training.

\section{Neurobiological Models of Mindfulness and Emotion Processing}

Neurobiological models of emotion processing describe a ventral "core affective" system responsible for establishing the threat or reward value of a stimulus, and a more dorsal affect processing system responsible for appraisals and attributions of one's emotional state (Barrett, Mesquita, Ochsner, \& Gross, 2007; Phillips, Drevets, Rauch, \& Lane, 2003). While regions of affective processing systems overlap somewhat, the ventral system for core affect has been described as including temporal lobe structures (including the amygdala), insula, anterior cingulate cortex (ACC), orbitofrontal cortex (OFC), and ventromedial prefrontal cortex (VMPFC). It is thought that this neuroanatomically coupled ventral network communicates the 
value of affective stimuli quickly and efficiently to hypothalamus and brainstem areas for coordinating a behavioral response (Barrett et al., 2007). The dorsal affect processing system is thought to be responsible for generating attributions about the cause(s) of one's core affective state, and includes the medial prefrontal cortex (MPFC), dorsomedial PFC (DMPFC) and ACC (Barrett et al., 2007). Finally, neurobiological models of emotion have also described the important role of PFC regions in the regulation of emotional states (Arnsten, 2009; Ochsner \& Gross, 2005). Specifically, these models indicate that explicit (e.g., cognitive reappraisal) and implicit (e.g., expectancies) emotion regulation strategies activate regions of dorsal and ventral PFC (including dorsal ACC), for modulating affect response regions (e.g., amygdala) (Ochsner \& Gross, 2005) (See Figure 1 for illustrations of these affective processing regions).

This review considers the evidence for mindfulness and emotional responding in light of these neurobiological models of emotion. The current research base shows that mindful attention and mindfulness training are implicated in modulating each of these neurobiological systems for emotional responding. Specifically, emerging research indicates that mindfulness can alter ventral neural regions for generating core affective responses, dorsal regions implicated in one's attributions about the cause of one's affective state, and in regions related to regulating one's affective responses.

\section{Measuring and Manipulating Mindfulness}

Mindfulness has been described as a process of paying attention "on purpose, in the present moment, and non-judgmentally” (Kabat-Zinn, 1994). A great deal of research has focused on operationally defining and measuring mindfulness, with varying perspectives and conceptualizations (for a review, see Brown, Ryan, \& Creswell, 2007; Lindsay, Creswell, \& Brown, in press). The scientific study of mindfulness has viewed mindfulness as both a 
dispositional quality that all individuals possess to varying degrees and an attentional state which can be fostered through training (Baer, 2003; Brown \& Ryan, 2003). Trait mindfulness has been measured by a variety of validated self-report questionnaires. In contrast to trait mindfulness, mindfulness training research entails training meditation naïve participants to adopt a mindful attentional stance while completing emotion tasks, or examining how brief (4 days to 10 -weeks) mindfulness meditation training impacts emotional responding. Finally, mindfulness training effects have been explored in studies that compare advanced meditators (with over 10 years of daily meditation practice, on average) to matched control participants. For a recent review of scientific measures and manipulations of mindfulness, see Lindsay et al. (in press).

For purposes of this chapter, we describe studies that include a measure or manipulation of mindfulness and a measure of brain activity while participants complete affective processing tasks. Accordingly, we first describe research relating dispositional (trait) mindfulness with neural measures of emotion processing. We then describe research exploring how a mindful attentional stance can impact neural markers of emotion processing. In the latter case, we order the sections by the amount of mindfulness training received: adopting a mindful attentional stance in meditation naïve participants, brief mindfulness meditation training, and in mindfulness meditation-trained experts.

\section{Trait Mindfulness and Emotion Processing}

Self-report measures of trait mindfulness have provided opportunities for investigators to relate self-reported individual differences in mindfulness to measures of brain activity during affective tasks. One recent study used electroencephalography (EEG) to assess the relationship between late positive potential (LPP) and trait mindfulness in an undergraduate sample (Brown, Goodman, \& Inzlicht, 2012). The LPP is a positive deflection of the event-related potential in 
the slow wave latency range ( $400-500 \mathrm{~ms}$ after stimulus onset), appearing most prominently in the posterior and central midline scalp regions. It is larger in response to more intense stimuli and correlates with subjective reports of arousal (Cuthbert, Schupp, Bradley, Birbaumer, \& Lang, 2000). Because of these characteristics, some researchers consider it a sensitive marker of early emotional arousal (Hajcak, MacNamara, \& Olvet, 2010). In this study of mindfulness and LPP response, researchers found that trait mindfulness as assessed by two self-report measures [the Mindful Attention Awareness Scale (Brown \& Ryan, 2003) and Five Facet Mindfulness Questionnare (Baer, Smith, Hopkins, Krietemeyer, \& Toney, 2006)] was associated with reduced LPP in response to high arousal, unpleasant stimuli (e.g., images of corpses). Trait mindfulness was also associated with reduced LPP in response to motivationally salient pleasant stimuli (e.g., erotica). These findings suggest that trait mindfulness is associated with a tempered early response $(\sim 500 \mathrm{~ms})$ to unpleasant and other motivationally salient stimuli that occurs before a subsequent response can arise and may indicate reduced emotional reactivity.

Another study assessed trait mindfulness with the Kentucky Inventory of Mindfulness Skills (KIMS; Baer, Smith, \& Allen, 2004) and asked participants to imagine personally experiencing emotional vignettes (Frewen et al., 2010). Using functional magnetic resonance imaging (fMRI), they found that greater self-reported individual differences in observing (on the Mindful Observing subscale) was positively associated with activation of the amygdala and DMPFC while listening to scripts designed to elicit experiences of rejection or social praise. The positive association between observing and amygdala activation is opposite to research showing that dispositional mindfulness is associated with reduced amygdala activation (Creswell, Way, Eisenberger, \& Lieberman, 2007; Modinos, Ormel, \& Aleman, 2010) (discussed below). Importantly, these studies that found an association between dispositional mindfulness and 
down-regulated amygdala activation used regulatory instructions to modify the response to affective stimuli, while the Frewen et al. study did not. Additionally, the observing subscale has been found to operate differently in meditating and non-meditating samples (Baer et al., 2008). In meditators, the observing subscale correlated with psychological adjustment and well-being. However, in non-meditators this subscale showed associations in the opposite direction. It may be that acceptance is an important underlying factor for mindfulness effects, specifically, an amygdala response may be higher when observing internal and external experiences without an accepting or nonjudgmental stance. The reported DMPFC activation found in the Frewen study associated with observing may be important for generating attributions about one's emotional state (Barrett et al., 2007) and may describe a potential neural underpinning of meta-cognitive awareness in mindful individuals (cf. Teasdale et al., 2002).

Two studies of trait mindfulness and emotion processing found mindfulness to be associated with increased PFC activity and reduced amygdala activity in response to affective stimuli (Creswell et al., 2007; Modinos et al., 2010). Both of these studies used regulatory experimental instructions while participants were viewing affective stimuli. Previous studies have shown that linguistically labeling affective images activates ventrolateral PFC (VLPFC), and deactivates the amygdala (Lieberman et al., 2007). This research suggesting that labeling one's feelings may be a basic mechanism for regulating one's emotions, and interestingly, labeling and noting are commonly used during mindfulness meditation practices (e.g., noting the experience of anger in the body). Building on this, Creswell and colleagues (2007) showed that dispositional mindfulness [as measured by the Mindful Attention Awareness Scale (MAAS; Brown \& Ryan, 2003)] moderated neural responses to an affect labeling task. Specifically, dispositional mindfulness was associated with greater activation of PFC regulatory regions 
(including bilateral VLPFC) and greater deactivation of the amygdala, suggesting that mindful individuals may be better able to recruit PFC regulatory regions during affect labeling. A similar neural affect regulation effect was observed in mindful individuals when instructed to use a cognitive reappraisal regulatory strategy. Modinos and colleagues (2010) asked participants to view negative images (e.g., burn victims, funeral scenes) and to reappraise, or reinterpret, their meaning so that it was no longer negative. They found that trait mindfulness (as measured by the KIMS) during reappraisal was associated with increases in DMPFC activity, and this activity was negatively correlated with amygdala activity.

\section{Summary: Trait Mindfulness Research}

Trait mindfulness research involving neural processing of affective stimuli suggests a relationship between trait mindfulness and reduced emotional reactivity. In studies using regulatory instructions, trait mindfulness is also associated with enhanced recruitment of emotion regulation regions. EEG research has linked dispositional mindfulness to reduced early cortical emotional reactivity (Brown, Goodman, \& Inzlicht, 2012). fMRI research using regulatory experimental instructions (e.g., affect labeling, cognitive reappraisal) showed that trait mindfulness is associated with increased PFC activation and reduced amygdala activation to affective images. Mindful traits were positively associated with increased activity in the PFC, and some functional connectivity findings (Creswell et al., 2007; Modinos et al., 2010) suggest that PFC is inversely associated with amygdala activity. This work suggests that when participants are given instructions to explicitly regulate affective responses (labeling, cognitive reappraisal), individual differences in mindfulness may activate emotion regulation regions in the PFC, which may in turn inhibit core affective responses in regions such as the amygdala. These findings suggesting a link between trait mindfulness and reduced emotional reactivity are 
important because emotional reactivity is central to dysfunctional emotion regulation (Linehan 1993a), and dysfuntion in emotion regulation is a core component in disorders of anxiety, mood, substance abuse, and eating (Berking \& Wupperman, 2012).

\section{State Mindfulness Research in Meditation Naïve Participants}

State mindfulness research can be categorized by participants' level of mindfulness training: meditation naïve, briefly trained, and expert. Studies of state mindfulness using meditation naïve participants instruct participants with no previous mindfulness training to adopt a mindful attentional stance. They use various experimental instructions to ask participants to pay attention to present moment experience. Instructions may ask participants to pay attention to present emotional experience and bodily sensations (Herwig, Kaffenberger, Jäncke, \& Brühl, 2010), or to actively monitor their responses to stimuli including thoughts, feelings, memories and body sensations with an accepting attitude (Westbrook et al., 2011).

Taylor and colleagues (2011) instructed meditation naïve participants to mindfully attend to images of differing emotional valences. These mindfulness instructions reduced self-reported emotional intensity experienced in response to the images across all valence categories (i.e., positive, negative $\&$ neutral). In these participants, a mindful attentional stance deactivated the amygdala in response to positive and negative images. Similarly, another study contrasted a narrative, conceptual think condition ("think about yourself, reflect who you are, about your goals") with a mindfulness of present moment emotions and bodily sensations feel condition in meditation naïve participants (Herwig et al., 2010). The think instructions increased amygdala activation, anterior cortical midline, and posterior cingulate cortex (PCC). The feel instructions deactivated the amygdala and resulted in a shift toward more posteriorly bilateral inferior frontal and premotor regions. The feel condition also activated the middle insula, which the authors 
interpreted as mindful attention increasing interoception (i.e., awareness of bodily states)

(Critchley, Wiens, Rotshtein, Öhman, \& Dolan, 2004).

One study examining mindfulness and cue-induced cigarette craving in meditation naïve participants also found that a mindful attentional stance reduces neural activity regions implicated in core affective reactivity (Westbrook et al., 2011). Most craving researchers categorize craving as an affective state for motivating behavior (see Skinner \& Aubin, 2010). In this study on mindfulness and craving, mindfulness instructions led to reduced self-reported cigarette craving and reduced neural reactivity to smoking cues in nicotine-deprived smokers. The ACC, including its subgenual region (sgACC), plays a central role in the craving response of dependent smokers (Kühn \& Gallinat, 2011). Mindfully attending to smoking cues not only reduced craving-related sgACC activation but also reduced its functional connectivity to other craving regions (e.g., ventral striatum). The area of deactivation around the sgACC extended to the ventromedial PFC (VMPFC), including Brodmann's area (BA) 10. BA 10 is thought to encode the subjective value of goods such as an appetitive snack (Hare, O'Doherty, Camerer, Schultz, \& Rangel, 2008). Mindfulness related reductions in this area may indicate a shift away from the subjective self-referential value of experience to a more objective, non-evaluative engagement with present moment experience.

In addition to reduction of emotional arousal, Farb and colleagues (2007) also showed a shift away from midline to lateral regions with engagement of a mindful attentional stance in meditation naïve participants. This study contrasted states of narrative focus and experiential focus while mildly positive (e.g., charming) and negative (e.g., greedy) characteristics were presented to meditation naïve participants undergoing fMRI. (While we discuss results obtained prior to mindfulness training here, we note that this study \& others that we review in this chapter 
included a mindfulness training component. We present neural results obtained after mindfulness training in our section on brief mindfulness training below.) Instructions for narrative focus entailed judging what is occurring, trying to figure out what that trait word means to the participant, whether it describes the participant, and allowing oneself to become caught up in a given train of thought. For experiential (mindfulness) focus, participants were to sense what is presently occurring in one's thoughts, feelings and body state, without a purpose or goal. Compared to narrative focus, experiential focus resulted in deactivation of cortical midline structures (subgenual cingulate, PCC, \& reduction in $\mathrm{mPFC}$ with lower threshold) and activation of a left-lateralized network (dorsolateral PFC, VLPFC, \& posterior parietal areas). The authors interpreted this to represent a shift away from the central default mode network (DMN) regions, the midline regions involved in narrative, self-referential processing. They interpreted reduced midline PFC activity as moving away from subjective, self-referntial valuation of experience to more objective and non-evaluative engagement (Farb et al., 2007).

\section{Summary: State Mindfulness Research with Meditation Nä̈ve Participants}

State mindfulness research using meditation naïve participants, as well as trait mindfulness findings, indicate a mindfulness-related decrease in core affective neural response and reported emotion reactivity (Farb et al., 2007; Herwig et al., 2010; Taylor et al., 2011; Westbrook et al., 2011). When engaging a mindful attentional stance, participants demonstrated deactivation of core affective, ventral regions and activation of dorsal and regulatory regions. As mentioned above, diminished emotional reactivity may allow for improved emotion regulation and thus discourage development of psychopathology. Instructions to engage a mindful attentional stance reduces reactivity and may therefore be protective. There is also some evidence to support a mindfulness-related shift away from the DMN and medial PFC toward a 
left lateralized network when participants engage a mindful (experiential) attentional focus (Farb et al., 2007; Herwig et al., 2010). Although more research is needed to evaluate this claim, these neural findings are consistent with the idea that a mindful attentional stance shifts one from a subjective, self-referential valuation of experience to a more objective and non-evaluative perception of experience. This non-evaluative perception of experience may be a mechanism whereby a mindful attentional stance reduces emotional reactivity. With non-evaluative perception, a stimulus loses the self-referential valence required for strong reactivity.

\section{State Mindfulness Research after Brief Mindfulness Meditation Training}

In contrast to instructing meditation naïve participants to adopt a mindful attentional stance, many studies offer participants a brief mindfulness meditation training program. A growing number of published studies randomly assign participants to brief mindfulness meditation training programs (or control programs) and compare changes in neural activation patterns to affective stimuli before and after this training. These training programs vary in length and intensity, ranging from four days of approximately 25 minutes of training per day (Zeidan et al., 2011), to 8 weeks of group and individual daily practice in variants of the Mindfulness-Based Stress Reduction (MBSR) program (Kabat-Zinn, 1994).

One recent study examined changes in neural processing of pain stimuli after a brief foursession 25-minute mindfulness training and compared this to an eyes closed rest condition (Zeidan et al., 2011). Participants were exposed to noxious heat stimuli while instructed to attend to the breath before and after mindfulness training. Attend to breath instructions did not reduce self-reported pain ratings before training, but it did after mindfulness training. Training also reduced activity in somatosensory cortex corresponding to the applied heat stimuli during the attend to breath condition. Participants who reported the greatest meditation-related 
reduction in pain intensity had the largest meditation-related activation of the anterior insula and ACC. With regard to pain, mindfulness training coupled with meditation instruction during application of pain stimuli results in reduced experienced pain. This reduction may be explained by the reduced activation of primary somatosensory cortex and increased activation of the anterior insula and ACC. The authors suggested that these effects may describe a neural basis for how mindfulness meditation alters appraisals that impart significance to salient sensory (pain) events (Zeidan et al., 2011), which is consistent with early mindfulness research showing that chronic pain patients had decoupled their pain sensations from their cognitive-affective reactions after MBSR training (Kabat-Zinn, 1982).

In contrast to this new research using quite brief 4-session mindfulness training, the MBSR program training consists of 8 weekly 2-hour group sessions, a 1-day silent retreat, and daily home meditation practice during the 8-week training program (Kabat-Zinn, 1994). This program is facilitated by a MBSR-trained instructor who maintains a daily mindfulness meditation practice. Mindfulness is taught through a progression of body-based mindfulness exercises (including guided meditations, mindful stretching and yoga, \& didactic exercises and group discussions). Two published studies examined differences in neural activation patterns after MBSR training while participants were exposed to emotional stimuli without any specific experimental instruction to modify their affective response. Both studies found reduced activity in the DMN and language areas in response to affective stimuli after mindfulness training. In the first study, participants diagnosed with social anxiety disorder (SAD) were shown positive and negative social trait adjectives before and after MBSR training and asked to consider if these traits described them (Goldin, Ramel, \& Gross, 2009). For positive traits, MBSR training resulted in reduced activity in self-referential DMN areas (medial PFC \& DMPFC) and language 
processing (left inferior frontal gyrus) areas. MBSR training increased activation to negative traits in visual attention areas (left inferior parietal lobule \& medial precuneus), possibly indicating reduced avoidance and increased ability to engage in negative social trait selfprocessing. Mindfulness training also resulted in decreased reported SAD symptoms (Goldin et al., 2009).

The second study exposed control and MBSR trained participants to sad film clips (Farb et al., 2010). Mindfulness training reduced neural activity in response to sad film clips compared to controls, particularly in the precuneus, $\mathrm{PCC}$, left posterior superior temporal gyrus (Wernicke's area), and left frontal operculum (Broca's area). The precuneus and PCC are midline cortical structures that have been associated with autobiographical memory retrieval and self-referential processing (Cavanna \& Trimble, 2006), and the PCC is a central node in the DMN. In addition to this shift away from DMN and language areas, MBSR participants showed more insula activity during sad clips than controls.

Another study that showed a mindfulness training-related reduction in DMN activity suggests that this reduction occurs with a decoupling of the DMN and insula (Farb et al., 2007). When experiential and narrative of focus were contrasted during presentation of positive and negative traits in participants who have completed MBSR, experiential focus reduced activity along the anterior cortical midline (rostral dorsal \& ventral $\mathrm{mPFC}$ ) and activates right regulatory PFC regions, insula, and secondary somatosensory cortex. Functional connectivity analyses showed that the insula was strongly correlated with VMPFC in controls but not in MBSR participants. Instead, the insula was coupled to DLPFC activity during experiential focus following meditation training. The authors suggested that interoception may be strongly coupled with narrative focus in controls but not in participants with mindfulness training. The overall 
pattern suggests that experiential (mindful) focus may reduce ventral core affective activity in the VMPFC and amygdala during the presentation of trait words, an effect that can be enhanced after mindfulness training. Moreover, mindfulness training increases the recruitment of rightlateralized PFC regulatory areas (right ventro- and dorsolateral PFC), providing suggestive evidence for mindfulness training effects on regulatory PFC regions (Cohen, Berkman, \& Lieberman, in press).

A recent study explored how MBSR training impacts neural responding in SAD patients. When these participants were instructed to shift attention to the breath while being exposed to negative self-beliefs, they exhibit a reduction in amygdala activity following MBSR training (Goldin \& Gross, 2010). However, when participants were exposed to negative self-beliefs without instructions to direct attention to the breath, there was an initial increase or spike of amygdala activity that quickly dissipated. Because these participants report reduced experienced negative emotion in response to negative self-beliefs, it may be that this initial spike in amygdala activity indicates that MBSR training increases initial affective orienting or emotional processing (Goldin \& Gross, 2010). Compared to baseline reacting to negative self-beliefs, these SAD participants also showed a shift away from anterior midline cortical and other DMN regions with training and breath focused attention. Similar to previous findings with SAD participants, they demonstrated increased activation of visual attention that may indicate reduced avoidance of negative stimuli.

In addition to the fMRI studies of brief mindfulness training, there are several studies that used EEG to examine the effects of brief mindfulness training on prefrontal $\alpha$-asymmetry. It is believed that anterior hemispheric asymmetry reflects motivational direction, with dominant lefthemispheric activity reflecting appetitive, approach responses and dominant right-hemispheric 
activity reflecting aversive, withdrawal responses (Davidson \& Irwin, 1999). EEG measurement of asymmetry in the $\alpha$-band $(8-13 \mathrm{~Hz})$ is used in this way to determine engagement of a positive, approach state or negative, withdrawal state. Studies that used this methodology have yielded mixed results. Two studies (one using MBSR \& another using an abbreviated 5-week training) found that mindfulness training shifted $\alpha$-asymmetry toward the left hemisphere suggesting that there may be a shift toward more approach-related positive emotionality (Davidson et al., 2003; Moyer et al., 2011); one study showed no change with meditation training and a shift toward right dominant asymmetry in controls using an 8-week MindfulnessBased Cognitive Therapy (MBCT) with participants with past suicidality (Barnhofer et al., 2007); and in one study the whole sample shifted toward right dominant $\alpha$-asymmetry regardless of whether participants were controls or mindfulness trained using MBCT (Keune, Bostanov, Hautzinger, \& Kotchoubey, 2011). One fMRI study not designed to assess $\alpha$-asymmetry noted dominant left PFC activity during meditation in expert meditators, and the authors interpreted this as indicative of a positive emotional state (Wang et al., 2011).

\section{Summary: State Mindfulness Research with Brief Mindfulness Meditation Training}

State mindfulness research involving brief mindfulness training and emotion processing indicates that training results in reduced markers of negative affect, such as SAD symptoms, negative emotion, and pain intensity and unpleasantness to an applied thermal pain probe (Goldin et al., 2009; Goldin \& Gross, 2010; Zeidan et al., 2011). These effects of brief mindfulness meditation training also co-occur with changes in specific neural activation patterns. Several studies indicate a mindfulness-related down-regulation of DMN areas (particularly the VMPFC, DMPFC, \& PCC) and language areas to a broad range of affective stimuli (Farb et al., 2007; Farb et al., 2010, Goldin et al., 2009; Goldin \& Gross 2010). This may indicate that brief 
mindfulness training shifts participants away from a self-referential, narrative focus and subjective valuation of experience. In response to affective stimuli, two studies showed overall mindfulness-related amygdala deactivation (Farb et al., 2007; Goldin \& Gross, 2010), and three studies found mindfulness increased insula activation (Farb et al., 2010; Farb et al., 2007; Zeidan et al., 2011). It may be that this insula activation indicates changes in interoception and the appraisal of salient sensory events. This body of work suggests that reduced negative affect as a result of mindfulness training may be driven by several underlying neural mechanisms: 1) deactivation of self-referential, evaluative, and narrative DMN regions 2) deactivation of the amygdala likely indicating reduced reactivity 3) increased insula activation indicative of altered interoception and representation of sensory events. These patterns indicate decreased activation of core affect regions both with and without the recruitment of affect regulation regions found in subjects high in trait mindfulness performing regulation tasks. It may be that a more objective perspective that accompanies movement away from self-referential DMN processing as a result of mindfulness training diminishes core affect reactivity without engaging regulatory processes. However, improved functioning of regulatory regions likely also accompanies mindfulness training. There are likely diverse neural pathways whereby mindfulness training can reduce negative affect in response to affective stimuli, and reduction of negative affect is critical to diverse clinical outcomes. EEG evidence, although mixed, suggests that brief mindfulness training may shift anterior hemispheric dominance to the left or prevent increases in right anterior dominance, which has been interpreted as promoting a more positive and approachoriented mental stance (Barnhofer et al., 2007; Davidson et al., 2003; Moyer et al., 2011). 
Another body of research examined functional neural differences between mindfulness practitioners with significant meditation experience (i.e., several years of daily practice) and meditation naïve controls matched on variables such as age, sex, education, and handedness (Brewer et al., 2011; Hölzel et al., 2007; Ives-Deliperi, Solms, \& Meintjes, 2011; Taylor et al., 2011; Wang et al., 2011). One study compared neural processing of emotionally evocative images in meditation naïve participants and meditators with over 1,000 hours of zen meditation experience under mindful viewing instruction and no instruction conditions (Taylor et al., 2011). When looking at images without viewing instructions, the only difference between beginning and experienced meditators is that experts had decreased activity in the rostro-ventral ACC when viewing positive images. Under mindful viewing instructions, both beginning and experienced meditators reported reduced emotional intensity experienced in response to the images with differing, group-specific neural correlates. Mindful instructions in beginners was associated with a deactivation of the amygdala during processing of positive and negative images. In experienced meditators, mindful viewing decreased activity in the medial PFC (BA 10) and PCC across all valence categories. In another study comparing experienced meditators to matched naïve controls while practicing different types of meditation, meditation in experienced meditators was associated with deactivation of the medial PFC and PCC (Brewer et al., 2011). Evidence suggests that mindfulness in experienced meditators entails a shift away from the DMN, including altered activation in the medial PFC (also part of the dorsal affective system).

\section{Our Emerging Understanding of Mindfulness and the Neurobiology of Emotion}

The findings to date indicate that mindfulness affects neurobiological networks implicated in emotion, including the ventral core affective network, the dorsal emotion processing network, and PFC regions implicated in the regulation of emotion. In the ventral core 
affective network, trait mindfulness and mindfulness training alter activation in amygdala, VMPFC, ACC, and insula in response to a broad range of affective stimuli. Reductions of amygdala activation in response to affective stimuli have been found in trait and state mindfulness studies (Creswell et al., 2007; Farb et al., 2007; Herwig, 2010; Modinos et al., 2010; Taylor et al., 2011). Along with other ventral affect processing regions (i.e., VMPFC \& ACC), the amygdala influences the visceromotor responses related to the value-based representations of an object (Barrett et al., 2007). Altered responses related to mindfulness in the VMPFC (Farb et al., 2007; Westbrook et al., 2011) and ACC (Farb et al., 2010; Taylor et al., 2011; Westbrook et al., 2011; Zeidan et al., 2011) have also been reported, also supporting the possibility of changes in visceromotor value-based responses associated with mindfulness.

These changes in this core affect response system coupled with a shift from midline DMN areas associated with self-referential valuation and narrative focus (i.e., VMPFC \& PCC) toward more lateral and posterior regions (Farb et al., 2010; Farb et al., 2007; Goldin et al., 2009; Goldin \& Gross, 2010) may indicate a shift away from subjective valuation and narrative elaboration toward a more experiential and objective awareness of present experience. Many theorists describe how mindfulness is characterized by a non-judgmental awareness of one's moment-to-moment experience (Kabat-Zinn, 1994), and this may reduce the evaluation of affective stimuli in terms of whether it is good or bad for "me" and reduce the elaboration of thoughts related to that evaluation. The increase in insula activity with mindfulness (Farb et al., 2010; Farb et al., 2007; Herwig et al., 2010; Taylor et al., 2011), and its decoupling from the valuation-related VMPFC (Farb et al., 2007) may also underlie the movement from subjective evaluation to a bare awareness of present experience. 
Mindfulness has also been linked with increase neural regulatory PFC regions when participants are instructed to regulate affect responses. When modifying responses to affective stimuli using a regulatory strategy (i.e, reappraisal or labeling), trait mindfulness is associated with increased PFC activation and decreased amygdala response (Creswell et al., 2007; Modinos et al., 2010). This suggests that individuals high in trait mindfulness might be better able to recruit regulatory networks when using a regulatory strategy. Changes in activation of the DMPFC, part of the PFC included in the dorsal affect processing system, are also related to mindfulness. This region may support attributions about affective experience. Three studies of trait mindfulness found mindfulness-related increases in DMPFC activity (Creswell et al., 2007; Frewen et al., 2010; Modinos et al., 2010), and one state mindfulness study in MBSR trained participants found a related decrease (Goldin et al., 2009). It may be that the DMPFC serves as part of the prefrontal regulatory system associated with regulatory strategies in individuals high in trait mindfulness and is down regulated during state mindfulness in the shift away from midline DMN areas.

In addition to the DMPFC activation mentioned above, mindfulness has also been found to be associated with altered activation of the MPFC and ACC, which have been described as emotion processing regions in the dorsal affect network (Barrett et al., 2007). In response to affective stimuli, a mindful attentional stance is associated with reduced activation of the MPFC (Farb et al., 2007; Goldin et al., 2009; Taylor et al., 2011). While the functional properties of the MPFC have yet to be precisely defined (Amodio \& Frith, 2006), it is thought that this region participates in attributions made about the cause(s) of core affect (Barrett et al., 2007). In contrast to the MPFC, the ACC has shown mindfulness-related activation in response to affective stimuli (Farb et al., 2010; Taylor et al., 2011; Zeidan et al., 2011), although craving related 
activation of the $\mathrm{sgACC}$ in response to smoking cues is reduced with mindfulness (Westbrook et al., 2011). The ACC is thought to signal the need to represent mental contents in consciousness with the aim of reducing conflict, improving understanding, or exerting greater control over them (Barrett et al., 2007). The pattern of increased ACC and decreased MPFC associated with mindfulness may indicate increased understanding and control of mental contents while deemphasizing attributions about the affect itself.

Our review has focused on describing findings from self-report measures of mindfulness and mindfulness training interventions, and future research would benefit from comparing how these different types of measures and manipulations of mindfulness relate to activation patterns in response to the same affective stimuli (e.g., a sad film clip known to elicit robust sadness) (cf., Goldin \& Gross, 2010; Taylor et al., 2011; Zeidan et al., 2011). When including state mindfulness, it would also be useful to ask participants how successful they felt they were in adopting a mindful attentional stance, and to include this in analyses. Instructions to engage mindful attention can be difficult to follow, and analyses using only subjects reporting success may further clarify mindful emotion processing patterns.

\section{Promising Question for Future Research}

How do mindfulness-related changes in neural emotion processing measures relate to changes in clinical symptoms?

Mindfulness-based interventions have been shown to reduce clinical symptoms of depression and anxiety (Hofmann et al., 2010; ; Roemer et al., 2008; Teasdale et al., 2000) as well as affective disturbances in chronic pain patients (Grossman et al., 2007; Kabat-Zinn, 1982; Kabat-Zinn et al., 1992). It seems likely that these clinical changes may be mediated by basic 
changes in neurobiological emotion processing systems, although very little work has attempted to explore neural mechanisms of these clinical symptom changes (Goldin et al., 2009; Goldin \& Gross, 2010). We are aware of several groups who are exploring these brain-behavior links, so we hope to see advances in this area in the coming years. One challenge in advancing research on this question is the complexity of analytic models for testing these mechanistic models, but advances in neuroimaging toolboxes for mediation analysis are now available (e.g., Wager et al., 2009).

How do mindfulness-related structural brain changes inform our understanding of mindfulness and emotion processing?

Several studies document mindfulness associated changes in gray matter density and volume in neural regions implicated in emotion processing (i.e., amygdala, hippocampus, and OFC). One study reported that MBSR reduced perceived stress, and reductions in perceived stress co-varied with decreases in gray matter density of the right amygdala (Hölzel et al., 2010). Given that some previous studies indicate that mindfulness alters amygdala response to affective stimuli (Creswell et al., 2007; Farb et al., 2007; Herwig et al., 2010; Modinos et al., 2010; Taylor et al., 2011), one promising future direction is to examine the relationship between structural and functional changes in amygdalar response (cf. Gianaros et al., 2008). Also, several studies indicate that mindfulness is associated with increases in grey matter density (Hölzel et al., 2011) and grey matter concentration (Hölzel et al., 2008; Luders, Toga, Lepore, \& Gaser, 2009) in the hippocampus. The hippocampus sits adjacent to amygdala and has been implicated as a core affective region. It is thought that hippocampus facilitates fear extinction, emotion processing, and memory (Corcoran, Desmond, Frey, \& Maren, 2005; Milad et al., 2007). Two studies indicate structural changes in orbitofrontal cortex (OFC) of experienced meditators (Hölzel et al., 
2008; Luders et al., 2009). Gray matter density in the medial OFC was positively associated with hours of meditation practice in experienced meditators (Hölzel et al., 2008). Another study found increased gray matter volumes in the OFC of experienced meditators compared to nonmeditators (Luders et al., 2009). The OFC, part of the ventral affective processing system, is thought to represent the affective value of an object in a flexible, experience- or contextdependent manner that the VMPFC uses to make choices and judgments based on this initial valuation (Barrett et al., 2007), suggesting that mindfulness training may increase processing capacity for considering contextual factors during emotion valuation.

How does mindful awareness impact neural affective responses over time?

Affective experiences are not monotonic; instead, they are content-rich events that arise and pass away over time. Accordingly, mindfulness has the potential to alter early orienting and attention toward affective stimuli (Jha, Krompinger, \& Baime, 2007; Vago \& Nakamura, 2011), to modify early emotion processing as affective stimuli are initially perceived (Brown et al., 2012), and to change how these stimuli are processed and regulated over time (Goldin \& Gross, 2010). We know very little about how mindful attention can alter this temporal process of emotion processing and its neural sequelae. For example, it is possible that mindful attention increases one's attention to threat related cues early in the emotion generation process, while also promoting a regulatory response during emotion processing. Indeed, an initial study suggests that this may be true (Vago \& Nakamura, 2011), although the neurobiological mechanisms are unknown. One limitation with current fMRI approaches is their sluggish temporal resolution (collecting a whole brain volume can take 1-3 seconds), which makes it difficult to evaluate changes in emotion processing to discrete affective events. Currently, EEG (Brown et al., 2012) 
and MEG (Kerr et al., 2011) approaches offer the best temporal resolution for testing these unexplored areas, and are exciting areas for future research.

\section{Conclusion}

An exciting body of research is emerging that identifies how mindfulness changes the way the brain processes affective stimuli. The body of work we describe here represents our first steps in understanding the neurobiology of mindfulness and emotion processing. Collectively, this initial work indicates that trait mindfulness and mindfulness-training can alter ventral core affective reactivity, dorsal emotion processing, and PFC regulatory neural affect regions. The coming years will no doubt bring new research that increases the specificity of our knowledge about the neurobiology of mindfulness and emotion processing. 


\section{References}

Amodio, D. M., Frith, C. D. (2006). Meeting of minds: the medial frontal cortex and social cognition. Nature Reviews Neuroscience, 7, 268-277.

Arnsten, A. F. T. (2009). Stress signalling pathways that impair prefrontal cortex structure and function. Nature Reviews Neuroscience, 10, 410-422.

Cohen, J. R., Berkman, E. T., \& Lieberman, M. D. (in press). Intentional and incidental selfcontrol in ventrolateral PFC. To appear in Oxford Handbook of Frontal Lobe Function.

Baer, R. A., Smith, G. T., \& Allen, K. B. (2004). Assessment of mindfulness by self-report. Assessment, 11, 191-206.

Baer, R. A., Smith, G. T., Hopkins, J., Krietemeyer, J., \& Toney, L. (2006). Using self-report assessment methods to explore facets of mindfulness. Assessment, 13, 27-45.

Baer, R. A., Smith, G. T., Lykins, E., Button, D., Krietemeyer, J., Sauer, S., . . Williams, J. M. G. (2008). Construct validity of the five facet mindfulness questionnaire in meditating and nonmeditating samples. Assessment, 15, 329-342.

Barnhofer, T., Duggan, D., Crane, C., Hepburn, S., Fennell, M. J., \& Williams, J. M. (2007). Effects of meditation on frontal alpha-asymmetry in previously suicidal individuals. Neuroreport, 18, 709-712.

Barrett, L. F., Mesquita, B., Ochsner, K. N., \& Gross, J. J. (2007). The experience of emotion. Annual Review of Psychology, 58, 373-403.

Berking, M., \& Wupperman, P. (2012). Emotion regulation and mental health: Recent findings, current challenges, and future directions. Current Opinion in Psychiatry, 25, 128-134. 
Brewer, J. A., Worhunsky, P. D., Gray, J. R., Tang, Y. Y., Weber, J., \& Kober, H. (2011). Meditation experience is associated with differences in default mode network activity and connectivity. Proceedings of the National Academy of Sciences, 108, 20254-20259.

Brown, K. W., Goodman, R. J., \& Inzlicht, M. (in press). Dispositional mindfulness and the attenuation of neural responses to emotional stimuli. Social Cognitive and Affective Neuroscience.

Brown, K. W., \& Ryan, R. M. (2003). The benefits of being present: mindfulness and its role in psychological well-being. Journal of Personality and Social Psychology, 84, 822-848.

Brown, K. W., Ryan, R. M., \& Creswell, J. D. (2007). Mindfulness: Theoretical foundations and evidence for its salutary effects. Psychological Inquiry, 18, 211-237.

Cavanna, A. E., \& Trimble, M. R. (2006). The precuneus: A review of its functional anatomy and behavioural correlates. Brain, 129, 564-583.

Corcoran, K. A., Desmond, T. J., Frey, K. A., \& Maren, S. (2005). Hippocampal inactivation disrupts the acquisition and contextual encoding of fear extinction. The Journal of Neuroscience, 25, 8978-8987.

Creswell, J. D., Way, B. M., Eisenberger, N. I., \& Lieberman, M. D. (2007). Neural correlates of dispositional mindfulness during affect labeling. Psychosomatic Medicine, 69, 560-565. Critchley, H. D., Wiens, S., Rotshtein, P., Öhman, A., \& Dolan, R. J. (2004). Neural systems supporting interoceptive awareness. Nature neuroscience, 7, 189-195.

Cuthbert, B. N., Schupp, H. T., Bradley, M. M., Birbaumer, N., \& Lang, P. J. (2000). Brain potentials in affective picture processing: Covariation with autonomic arousal and affective report. Biological Psychology, 52, 95-111. 
Davidson, R. J., \& Irwin, W. (1999). The functional neuroanatomy of emotion and affective style. Trends in Cognitive Sciences, 3, 11-21.

Davidson, R. J., Kabat-Zinn, J., Schumacher, J., Rosenkranz, M., Muller, D., Santorelli, S. F., . . . Sheridan, J. F. (2003). Alterations in brain and immune function produced by mindfulness meditation. Psychosomatic Medicine, 65, 564-570.

Farb, N. A. S., Anderson, A. K., Mayberg, H., Bean, J., McKeon, D., \& Segal, Z. V. (2010). Minding one's emotions: Mindfulness training alters the neural expression of sadness. Emotion, 10, 25-33.

Farb, N. A. S., Segal, Z. V., Mayberg, H., Bean, J., McKeon, D., Fatima, Z., \& Anderson, A. K. (2007). Attending to the present: Mindfulness meditation reveals distinct neural modes of self-reference. Social Cognitive and Affective Neuroscience, 2, 313-322.

Frewen, P. A., Dozois, D. J. A., Neufeld, R. W. J., Lane, R. D., Densmore, M., Stevens, T. K., \& Lanius, R. A. (2010). Individual differences in trait mindfulness predict dorsomedial prefrontal and amygdala response during emotional imagery: An fMRI study. Personality and Individual Differences, 49, 479-484.

Gianaros, P. J., Sheu, L. K., Matthews, K. A., Jennings, J. R., Manuck, S. B., \& Hariri, A. R. (2008). Individual differences in stressor-evoked blood pressure reactivity vary with activation, volume, and functional connectivity of the amygdala. The Journal of Neuroscience, 28, 990-999.

Goldin, P., Ramel, W., \& Gross, J. (2009). Mindfulness meditation training and self-referential processing in social anxiety disorder: Behavioral and neural effects. Journal of Cognitive Psychotherapy, 23, 242-257. 
Goldin, P. R., \& Gross, J. J. (2010). Effects of mindfulness-based stress reduction (MBSR) on emotion regulation in social anxiety disorder. Emotion, 10, 83-91.

Grossman, P., Tiefenthaler-Gilmer, U., Raysz, A., \& Kesper, U. (2007). Mindfulness training as an intervention for fibromyalgia: Evidence of postintervention and 3-year follow-up benefits in well-being. Psychotherapy and Psychosomatics, 76, 226-233.

Hajcak, G., MacNamara, A., \& Olvet, D. M. (2010). Event-related potentials, emotion, and emotion regulation: An integrative review. Developmental Neuropsychology, 35, 129155.

Hare, T. A., O'Doherty, J., Camerer, C. F., Schultz, W., \& Rangel, A. (2008). Dissociating the role of the orbitofrontal cortex and the striatum in the computation of goal values and prediction errors. The Journal of Neuroscience, 28, 5623-5630.

Herwig, U., Kaffenberger, T., Jäncke, L., \& Brühl, A. B. (2010). Self-related awareness and emotion regulation. Neuroimage, 50, 734-741.

Hofmann, S. G., Sawyer, A. T., Witt, A. A., \& Oh, D. (2010). The effect of mindfulness-based therapy on anxiety and depression: A meta-analytic review. Journal of Consulting and Clinical Psychology, 78, 169-183.

Hölzel, B. K., Carmody, J., Evans, K. C., Hoge, E. A., Dusek, J. A., Morgan, L., . . Lazar, S. W. (2010). Stress reduction correlates with structural changes in the amygdala. Social Cognitive and Affective Neuroscience, 5, 11-17.

Hölzel, B. K., Carmody, J., Vangel, M., Congleton, C., Yerramsetti, S. M., Gard, T., \& Lazar, S. W. (2011). Mindfulness practice leads to increases in regional brain gray matter density. Psychiatry Research: Neuroimaging, 191, 36-43. 
Hölzel, B. K., Ott, U., Gard, T., Hempel, H., Weygandt, M., Morgen, K., \& Vaitl, D. (2008). Investigation of mindfulness meditation practitioners with voxel-based morphometry. Social Cognitive and Affective Neuroscience, 3, 55-61.

Hölzel, B. K., Ott, U., Hempel, H., Hackl, A., Wolf, K., Stark, R., \& Vaitl, D. (2007).

Differential engagement of anterior cingulate and adjacent medial frontal cortex in adept meditators and non-meditators. Neuroscience Letters, 421, 16-21.

Ives-Deliperi, V. L., Solms, M., \& Meintjes, E. M. (2011). The neural substrates of mindfulness: An fMRI investigation. Social Neuroscience, 6, 231-242.

Jha, A. P., Krompinger, J., \& Baime, M. J. (2007). Mindfulness training modifies subsystems of attention. Cognitive, Affective, \& Behavioral Neuroscience, 7, 109-119.

Kabat-Zinn, J. (1982). An outpatient program in behavioral medicine for chronic pain patients based on the practice of mindfulness meditation: Theoretical considerations and preliminary results. General hospital psychiatry, 4, 33-47.

Kabat-Zinn, J. (1994). Wherever you go, there you are: Mindfulness meditation in everyday life. New York: Hyperion Books.

Kabat-Zinn, J., Massion, A. O., Kristeller, J., Peterson, L. G., Fletcher, K. E., Pbert, L., .. . Santorelli, S. F. (1992). Effectiveness of a meditation-based stress reduction program in the treatment of anxiety disorders. The American Journal of Psychiatry, 149, 936-943.

Kerr, C. E., Jones, S. R., Wan, Q., Pritchett, D. L., Wasserman, R. H., Wexler, A., .. Kaptchuk, T. J. (2011). Effects of mindfulness meditation training on anticipatory alpha modulation in primary somatosensory cortex. Brain Research Bulletin, 84, 96-103. 
Keune, P. M., Bostanov, V., Hautzinger, M., \& Kotchoubey, B. (2011). Mindfulness-based cognitive therapy (MBCT), cognitive style, and the temporal dynamics of frontal EEG alpha asymmetry in recurrently depressed patients. Biological Psychology, 88, 243-253.

Kühn, S., \& Gallinat, J. (2011). Common biology of craving across legal and illegal drugs-a quantitative meta-analysis of cue-reactivity brain response. European Journal of Neuroscience, 33(7), 1318-1326.

Lieberman, M. D., Eisenberger, N. I., Crockett, M. J., Tom, S. M., Pfeifer, J. H., \& Way, B. M. (2007). Putting feelings into words affect labeling disrupts amygdala activity in response to affective stimuli. Psychological Science, 18, 421-428.

Lindsay, E.K., Creswell, J.D., \& Brown, K.W. (in press). Conceptualizations, measures, and manipulations of mindfulness. Eds. Brown, K.W., Creswell, Ryan, R. Handbook on Mindfulness Science. New York: Guilford Publications.

Linehan, M. (1993). Cognitive-behavioral treatment of borderline personality disorder. New York: Guilford Press.

Luders, E., Toga, A. W., Lepore, N., \& Gaser, C. (2009). The underlying anatomical correlates of long-term meditation: Larger hippocampal and frontal volumes of gray matter. Neuroimage, 45, 672-678.

Milad, M. R., Wright, C. I., Orr, S. P., Pitman, R. K., Quirk, G. J., \& Rauch, S. L. (2007). Recall of fear extinction in humans activates the ventromedial prefrontal cortex and hippocampus in concert. Biological Psychiatry, 62, 446-454.

Modinos, G., Ormel, J., \& Aleman, A. (2010). Individual differences in dispositional mindfulness and brain activity involved in reappraisal of emotion. Social Cognitive and Affective Neuroscience, 5, 369-377. 
Moyer, C. A., Donnelly, M. P. W., Anderson, J. C., Valek, K. C., Huckaby, S. J., Wiederholt, D. A., ... Rice, B. L. (2011). Frontal electroencephalographic asymmetry associated with positive emotion is produced by very brief meditation training. Psychological Science, $22,1277-1279$.

Ochsner, K. N., \& Gross, J. J. (2005). The cognitive control of emotion. Trends in cognitive sciences, 9, 242-249.

Phillips, M. L., Drevets, W. C., Rauch, S. L., \& Lane, R. (2003). Neurobiology of emotion perception I: The neural basis of normal emotion perception. Biological psychiatry, 54, 504-514.

Roemer, L., Orsillo, S. M., \& Salters-Pedneault, K. (2008). Efficacy of an acceptance-based behavior therapy for generalized anxiety disorder: Evaluation in a randomized controlled trial. Journal of Consulting and Clinical Psychology, 76, 1083-1089.

Skinner, M. D., \& Aubin, H. J. (2010). Craving's place in addiction theory: Contributions of the major models. Neuroscience and Biobehavioral Reviews, 34, 606-623.

Taylor, V., Grant, J., Daneault, V., Scavone, G., Breton, E., Roffe-Vidal, S., . . Beauregard, M. (2011). Impact of mindfulness on the neural responses to emotional pictures in experienced and beginner meditators. Neuroimage, 57, 1524-1533.

Teasdale, J. D., Moore, R. G., Hayhurst, H., Pope, M., Williams, S., \& Segal, Z. V. (2002). Metacognitive awareness and prevention of relapse in depression: Empirical evidence. Journal of Consulting and Clinical Psychology, 70, 275-287.

Teasdale, J. D., Segal, Z. V., Williams, J. M. G., Ridgeway, V. A., Soulsby, J. M., \& Lau, M. A. (2000). Prevention of relapse/recurrence in major depression by mindfulness-based cognitive therapy. Journal of Consulting and Clinical Psychology, 68, 615-623. 
Vago, D. R., \& Nakamura, Y. (2011). Selective attentional bias towards pain-related threat in fibromyalgia: preliminary evidence for effects of mindfulness meditation training. Cognitive Therapy and Research, 35, 1-14.

Wager, T. D., van Ast, V. A., Hughes, B. L., Davidson, M. L., Lindquist, M. A., \& Ochsner, K. N. (2009). Brain mediators of cardiovascular responses to social threat, part II:

Prefrontal-subcortical pathways and relationship with anxiety. Neuroimage, 47, 836-851.

Wang, D. J. J., Rao, H., Korczykowski, M., Wintering, N., Pluta, J., Khalsa, D. S., \& Newberg, A. B. (2011). Cerebral blood flow changes associated with different meditation practices and perceived depth of meditation. Psychiatry Research: Neuroimaging, 191, 60-67.

Westbrook, C., Creswell, J. D., Tabibnia, G., Julson, E., Kober, H., \& Tindle, H. A. (in press). Mindful attention reduces neural and self-reported cue-induced craving in smokers. Social Cognitive and Affective Neuroscience.

Zeidan, F., Martucci, K. T., Kraft, R. A., Gordon, N. S., McHaffie, J. G., \& Coghill, R. C. (2011). Brain mechanisms supporting the modulation of pain by mindfulness meditation. The Journal of Neuroscience, 31, 5540-5548. 

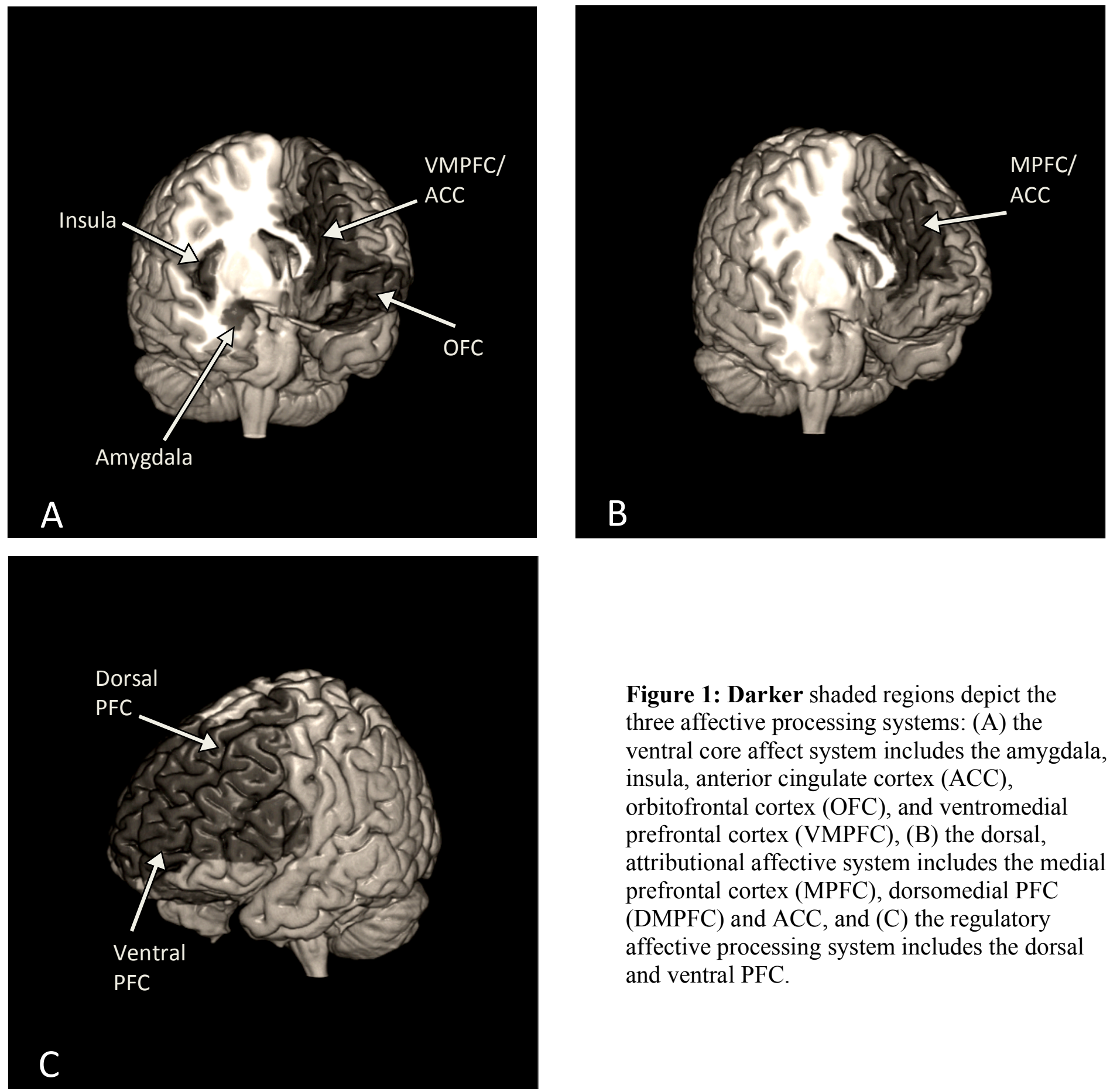

Figure 1: Darker shaded regions depict the three affective processing systems: (A) the ventral core affect system includes the amygdala, insula, anterior cingulate cortex (ACC), orbitofrontal cortex (OFC), and ventromedial prefrontal cortex (VMPFC), (B) the dorsal, attributional affective system includes the medial prefrontal cortex (MPFC), dorsomedial PFC (DMPFC) and ACC, and $(\mathrm{C})$ the regulatory affective processing system includes the dorsal and ventral PFC. 